\title{
MicroRNA-302b-3p Suppresses Cell Proliferation Through AKT Pathway by Targeting IGF-1R in Human Gastric Cancer
}

\author{
Bo Guo $^{\mathrm{a}}$ Zhenghao Zhao ${ }^{\mathrm{b}}$ Zhen Wang ${ }^{\mathrm{a}}$ Qian Li $^{\mathrm{a}} \quad$ Xiaofei Wang ${ }^{\mathrm{a}}$ Wenjing Wang ${ }^{\mathrm{c}}$ \\ Tusheng Song ${ }^{\text {a }}$ Chen Huang a,d,e \\ aDepartment of cell Biology and Genetics, School of Basic Medical Sciences, Xi'an Jiaotong University

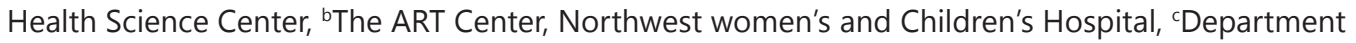 \\ of Hepatobiliary Surgery, First Affiliated Hospital of Xi'an Jiaotong University, 'Key Laboratory of \\ Shaanxi Province for Craniofacial Precision Medicine Research, Stomatology College of Xi'an Jiaotong \\ University, ${ }^{e}$ Key Laboratory of Environment and Genes Related to Diseases, Xi'an Jiaotong University, \\ Ministry of Education of China, Xi'an, China
}

\section{Key Words}

MiR-302b-3p • Gastric cancer • IGF-1R • Proliferation • AKT signaling pathway

\begin{abstract}
Background/Aims: MiR-302b is a major microRNA found in human embryonic stem cells and induced pluripotent stem cells. However, its function in gastric cancer progression remains unclear. Methods: Quantitative reverse transcription-PCR was performed to detect the expression levels of miR-302b-3p in gastric cancer tissues. MTT, colony formation, and flow cytometer analyses were conducted to explore the function of miR-302b-3p in MKN-45/ SGC-7901 cells. A dual-luciferase reporter was used to validate the bioinformatics-predicted target gene of miR-302b-3p. Western blotting and RNA interference were used to evaluate the expression of the AKT signaling pathway and determine the mechanisms underlying miR-302b-3p-induced anti-tumor effects. Results: MiR-302b-3p expression was decreased in gastric cancer tissues and cell lines. Enforced expression of miR-302b suppressed cell proliferation and cell cycle G1-S transition and induced apoptosis. IGF-1R was found to be a direct target of miR-302b-3p, and silencing of IGF-1R resulted in the same biological effects as those induced by miR-302b-3p overexpression in gastric cancer cells. Importantly, both overexpression of miR-302b-3p and silencing of IGF-1R decreased AKT phosphorylation, which modulated AKT related cell cycle regulators (cyclin A2, cyclin D1, CDK2, and CDk6) and apoptotic protein $\mathrm{Bax} / \mathrm{Bcl}-2$. Conclusion: These results indicate the tumor suppressor role of miR-302b-3p in the pathogenesis of gastric cancer.




\section{Cellular Physiology Cell Physiol Biochem 2017;42:1701-1711 \begin{tabular}{l|l} 
DOI: 10.1159/000479419 & O 2017 The Author(s). Published by S. Karger AG, Basel \\
www.karger.com/cpb
\end{tabular} Guo et al.: MiR-302b-3p Targets IGF-1R in Gastric Cancer SGC-7901/MKN-45 Cells}

\section{Introduction}

Gastric cancer (GC) is one of the most frequent malignancies and second most common cause of death from cancer worldwide [1]. The development and progression of GC is thought to be a multistep process involving the accumulation of several genetic mutations and alterations in proto-oncogenes or tumor-suppressor genes [2, 3]. Unfortunately, most patients with GC have poor prognosis because this cancer is typically diagnosed at a late stage of disease. Therefore, further insight into the molecular mechanisms underlying GC progression may identify novel therapeutic targets and improve the prognosis of GC.

MicroRNAs (miRNAs) are endogenous noncoding RNAs of 19-24 nucleotides in length, which could regulate hundreds of target genes by binding to the $3^{\prime}$ untranslated region (UTR) that may result in mRNA degradation or translational repression [4]. Aberrant expression of miRNAs has been well-described in many types of tumors, including leukemia [5], breast [6], prostate [7], and ovarian [8] cancers. Numerous miRNAs associated with GC have been identified. Recently, we and others have identified some miRNAs that are deregulated in GC, such as down-regulation of miR-338-3p [9], miR-429 [10], and miR-449a [11] and upregulation of miR-21 [12] and miR-106a [13]. In this study, we found that miR-302b-3p is one of the most frequently down-regulated miRNAs in GC tissues and cell lines. MiR-302b functions as a tumor-suppressor in different types of cancers, include in lung [14], liver [15], and colon cancer [16]. The role of miR-302b-3p and its underlying mechanisms in GC remain unclear.

Here, we found that miR-302b-3p significantly inhibits GC SGC-7901/MKN-45 cell proliferation through the AKT pathway by targeting insulin-like growth factor 1 receptor (IGF-1R) via its 3' UTR. Furthermore, miR-302b-3p induced SGC-7901/MKN-45 cell cycle transition arrest in the G1-S phase, promoted cell apoptosis, and subsequently interrupted AKT-related cell cycle and apoptotic regulators. Silencing of IGF-1R had similar cellular and molecular effects as miR-302b-3p overexpression had. These finding reveal the tumorsuppressor roles of miR-302b-3p in GC progression, which may be used to develop a beneficial strategy for future cancer therapy.

\section{Materials and Methods}

\section{Human tissue samples and cell lines}

The immortalized gastric epithelial cell lines (GES-1) and human gastric cancer cell lines (SGC-7901 and MKN-45) were maintained in the Key Laboratory of Environment and Genes Related to Diseases at Xi'an Jiaotong University. Cells were cultivated in Dulbecco's Modified Eagle Medium (PAA Laboratories GmbH, Etobicoke, Ontario, Canada) supplemented with $10 \%$ fetal bovine serum (PAA Laboratories $\mathrm{GmbH}$ ) at $37^{\circ} \mathrm{C}$ in a humidified atmosphere of $95 \%$ air and $5 \% \mathrm{CO}_{2}$. Thirty-eight paired $\mathrm{GC}$ and adjacent non-tumor gastric tissues were obtained from patients who had undergone surgical gastric resection at the First Affiliated Hospital of Xi'an Jiaotong University. Informed consent was obtained from each patient, and the study was approved by the Institute Research Ethics Committee at Cancer Center, Xi'an Jiaotong University. No local or systemic treatment had been conducted before operation. Tissue samples were immediately frozen in liquid nitrogen until RNA extraction.

Quantitative real-time reverse transcription ( $q R T) P C R$

Total RNA was extracted from the cells or tissues with TRIzol reagent (Invitrogen, Carlsbad, CA, USA). PrimeScript RT Reagent Kit (TAKARA, Shiga, Japan) and SYBR Premix Ex Taq II Kit (TAKARA) were used to detect miR-302b-3p and IGF-1R expression according to the manufacturer's instructions. The following primers were used: (IGF-1R-F: 5'-TTTCCCACAGCAGTCCACCTC-3', IGF-1R-R: 5'-AGCATCCTAGCCTTCTCACCC-3'; $\beta$-actin-F: $\quad$ 5'-CCAACCGCGAGAAGATGA-3', $\quad \beta$-actin-R: $\quad$ 5'-CCAGAGGCGTACAGGGATAG-3'; miR-302b3p-RT: 5'-GTCGTATCCAGTGCGTGTCGTGGAGTCGGCAATTGCACTGGATACGACCTACTAA-3', miR302b-3p-F: 5'-ATCCAGTGCGTGTCGTG-3', miR-302b-3p-R: 5'-TGCTTAAGTGCTTCCATGTT-3'; U6RT: 5'-CGCTTCACGAATTTGCGTGTCAT-3', U6-F: 5'-GCTTCGGCAGCACATATACTAAAAT-3', U6-R: 


\section{Cellular Physiology Cell Physiol Biochem 2017;42:1701-1711 \begin{tabular}{ll|l} 
and Biochemistry & $\begin{array}{l}\text { DOI: 10.1159/000479419 } \\
\text { Published online: July 25, } 2017\end{array}$ & $\begin{array}{l}\text { @ } 2017 \text { The Author(s). Published by S. Karger AG, Basel } \\
\text { www.karger.com/cpb }\end{array}$ \\
\hline
\end{tabular}

5'-CGCTTCACGAATTTGCGTGTCAT-3'). The $2^{\text {-DDCt }}$ method was used with the RNU6B (U6) gene as a control for miRNA, while the $\beta$-actin gene was used as a control for mRNAs. All reactions were performed in triplicate using the IQ-5 Real-Time PCR System (Bio-Rad, Hercules, CA, USA).

\section{Expression vector construction}

The miR-302b-3p expression vector was constructed with synthetic oligonucleotides and cloned between the EcoRI and HindIII sites of the pcDNATM6.2-GW/EmGFP-miR vector (Invitrogen). The 3' UTR of human IGF-1R was constructed using synthetic oligonucleotides and cloned between the SacI and XhoI sites of the pmirGLO Dual-Luciferase miRNA Target Expression Vector (Promega, Madison, WI, USA). The inhibitor of miR-302b-3p (anti-miR-302b-3p) and small interfering RNA (siRNA) targeting IGF-1R (si-IGF$1 \mathrm{R})$ were purchased from Gene-Pharma (Shanghai, China). All vector sequence information is as follows: (pre-miR-302b sense: 5'-AAT TCG CTC CCT TCA ACT TTA ACA TGG AAG TGC TTT CTG TGA CTT TAA AAG TAA

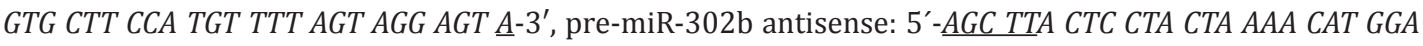
AGC ACT TAC TTT TAA AGT CAC AGA AAG CAC TTC CAT GTT AAA GTT GAA GGG AGC G-3'; IGF-1R-3'UTR-WTsense: 5'-CAC ACT TAA TAG CAA CAG AGC ACT TGL-3', IGF-1R-3'UTR-WT-antisense: 5'-TCG AGC AAG TGC TCT

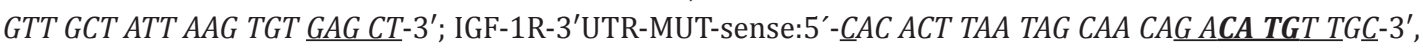
IGF-1R-3'UTR-MUT-antisense: 5'-TCG AGC AAC ATG TCT GTT GCT ATT AAG TGT GAG CT-3'.

\section{Bioinformatic analysis}

High-throughput expression analysis of IGF-1R was performed with The Cancer Genome Atlas (TCGA) database and information for human miR-302b-3p was registered and obtained in miRBase. miRNA targets were predicted using 3 publicly available programs: TargetScan, miRanda, and PicTar.

\section{Dual-luciferase assay}

The IGF-1R-3' UTR-wt vector or IGF-1R-3' UTR-mut vector were co-transfected with miR-302b-3p into the HEK293 cell lines, while the pmirGLO vector was used as a control. Reporter gene assays were performed $24 \mathrm{~h}$ later using the Dual Luciferase Reporter assay system (Promega). Normalized firefly luciferase activity was obtained by firefly luciferase activity/Renilla luciferase activity according to the manufacturer's protocol. All experiments were performed at least three times.

\section{Cell-proliferation assay}

Cells (5000 cells/well) were seeded into 96-well plates with $100 \mu \mathrm{L}$ of DMEM and incubated for $24 \mathrm{~h}$. Next, 0.2 mg of DNA plasmid or siRNA was transfected into the cells and the cells were further cultivated for an additional 1-3 days. Cell viability was assessed using the MTT assay on FLUOstar OPTIMA (BMG Labtech, Offenburg, Germany). Each experiment contained 3 replicates and was repeated at least twice. The data are summarized as the mean $\pm \mathrm{SD}$.

\section{Cell colony formation assay}

Stably transfected cells were seeded at a density of $5 \times 10^{3}$ per 6-well plate. After incubation for 1-2 weeks, cell colonies were stained with $0.5 \%$ crystal violet for $30 \mathrm{~min}$. The stained cell clones were photographed after excess dye was rinsed off twice with PBS, and the colony number ( $>10$ cells) was recorded to calculate cell cloning efficiency.

\section{Cell apoptosis analysis}

Cell apoptosis analysis was performed with the Annexin-V FITC Apoptosis Detection Kit (Invitrogen) according to the manufacturer's instructions. The cells were seeded into 12-well plates at a density of $1 \times$ $10^{6}$ cells per well in triplicate and transfected with DNA plasmid or siRNAs for $24 \mathrm{~h}$, and then examined by flow cytometry (BD Biosciences, Franklin Lakes, NJ, USA). Apoptosis populations were determined using ModFit software.

Cell cycle analysis

Cells at $1 \times 10^{6}$ cells per well were cultured in 12-well plates in triplicate and transfected for $24 \mathrm{~h}$. The cells were harvested by trypsinization and fixed in ice-cold ethanol overnight at $4^{\circ} \mathrm{C}$. The cells were then washed in PBS and incubated in $300 \mu \mathrm{L}$ of staining solution $(20 \mathrm{mg} / \mathrm{mL}$ propidium iodide and $10 \mathrm{U} / \mathrm{mL}$ 


\section{Cellular Physiology Cell Physiol Biochem 2017;42:1701-1711 and Biochemistry Published online: July 25, $2017 \quad \begin{aligned} & \text { DOI: 10.1159/000479419 } 2017 \text { The Author(s). Published by S. Karger AG, Basel } \\ & \text { www.karger.com/cpb }\end{aligned}$

RNaseA) for $30 \mathrm{~min}$ at room temperature. Cell-cycle distributions were assayed by fluorescence-activated cell sorting based on flow cytometer.

\section{Western blot analysis}

Total protein was extracted with lysis buffer (Wolsen, Xi'an, China) from cells harvested $48 \mathrm{~h}$ after transfection, separated in $10 \%$ SDS polyacrylamide gels, and electrophoretically transferred to polyvinylidene difluoride membranes (Millipore, Billerica, MA, USA). Mouse polyclonal anti-IGF-1R (1:1000, Cell Signaling Technology, Danvers, MA, USA), rabbit mAb anti-Akt, anti-phospho-Akt, anti-Bax and anti-Bcl-2 (1:1000, Cell Signaling Technology), rabbit mAb anti-cyclin D1, anti-cyclin A2, anti-CDK2, anti-CDK6 (1:500, Bioworld Technology), and mouse monoclonal anti- $\beta$-actin antibody (1:2000; Cell Signaling Technology) were used for detection with an enhanced chemiluminescence detection system (Pierce, Rockford, IL, USA). The blots were scanned and band density was measured using Quantity One imaging software.

\section{Statistical analysis}

Each experiment was repeated at least 3 times independently. The data were presented as the mean \pm SD and analyzed using PASW Statistics 18 software (SPSS, Inc., Chicago, IL, USA). Differences or correlations between 2 groups were calculated by Student's $t$-test or Pearson correlation. $\mathrm{P} \leq 0.05$ was considered significant.

\section{Results}

Aberrant miR-302b-3p expression in human GC

To validate the role of miR-302b-3p in GC, we analyzed the expression in 20 pairs of GC tissue and matched adjacent normal tissue samples by real-time PCR. Compared with normal tissues, significant down-regulation of miR-302b-3p was observed in 85\% (17 of 20) of GC samples (Fig. 1A). Next, we found that miR-302b-3p was down-regulated in the GC cell lines compared to in gastric epithelial cell lines (Fig. 1B). This observation was consistent with the results for the expression of miR-302b-3p in GC tissues at the RNA level. We examined the correlation between miR-302b-3p levels and clinicopathological factors, but the results were not significant, possibly because of the limited sample size (data not shown). These data suggest that miR-302b-3p functions as a tumor-suppressor in gastric cancer.

\section{IGF-1R is a direct target of $\mathrm{miR}-302 \mathrm{~b}-3 p$}

We searched for miR-302b-3p target genes using three computer-aided miRNA target prediction programs: TargetScan, PicTar, and miRanda. We found a well-matched miR-302b$3 p$ binding site in the IGF-1R 3' UTR. Comparison of the human sequence with other species revealed that the targeting sequence was highly conserved among different species (Fig. 2A). To validate the hypothesis that IGF-1R is a target of miR-302b-3p, a dual-luciferase reporter system containing IGF-1R-3' UTR-wt and IGF-1R-3' UTR-mut was used. HEK293 cells were cotransfected with miR-302b-3p and a reporter plasmid or pmirGLO control vector. As a result, miR-302b-3p clearly suppressed the firefly luciferase activity of IGF-1R-3' UTR-wt at $24 \mathrm{~h}$

Fig. 1. MiR-302b-3p is down-regulated in gastric cancer tissues and cell lines. (A) qRT-PCR was performed to examine miR-302b$3 p$ expression in 20 paired human gastric cancer and adjacent normal tissues. (B) qRT-PCR analysis of miR-302b-3p expression in normal gastric and gastric cancer cells. The expression of $\mathrm{miR}-302 \mathrm{~b}-3 \mathrm{p}$ was

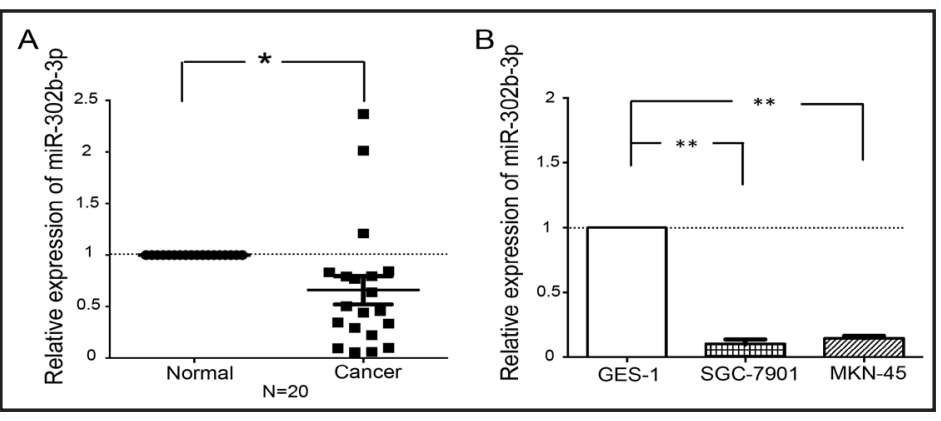
normalized against an endogenous control (U6 RNA). Data were analyzed using a $2^{-\Delta \Delta \mathrm{Ct}}$ approach. All data are shown as mean \pm SD for three independent experiments $\left(P^{*}<0.05, P^{* *}<0.01\right)$. 
Fig. 2. IGF-1R is experimentally validated as a direct target of miR-302b-3p in gastric cancer cells. (A) MiR-302b-3p is highly conserved across species and binding sites within seed region sequence in the 3'-UTR of human IGF-1R. (B) Luciferase assay in HEK293 cells. pmirGLOIGF-1R-wt or pmirGLO-IGF-1R-mt vector was co-transfected with miR-302b-3p vector. Luciferase activity in pmirGLOIGF-1R-wt group denoted a statistically significant decrease following ectopic expression of miR-302b-3p. (C/D) IGF$1 \mathrm{R}$ expression level was measured after transfection with miR-302b-3p vector or anti-miR-302b-3p in SGC-7901/MKN-45 cells. (E) mRNA and protein expression of IGF-1R were determined by qRT-PCR and Western blot in GC tissues and their corresponding normal tissues selected randomly. (F) IGF-1R expression was determined by qRT-PCR and Western blot in gastric cancer cell lines and immortalized gastric epithelial cell lines. (G) IGF1R shown no statistic difference in 405 cancer tissues compared to 35 normal tissues as well as an elevated expression of IGF-1R was found in 26 paired gastric clinical tissues. $\left(P^{*}<0.05, P^{* *}<0.01\right)$.

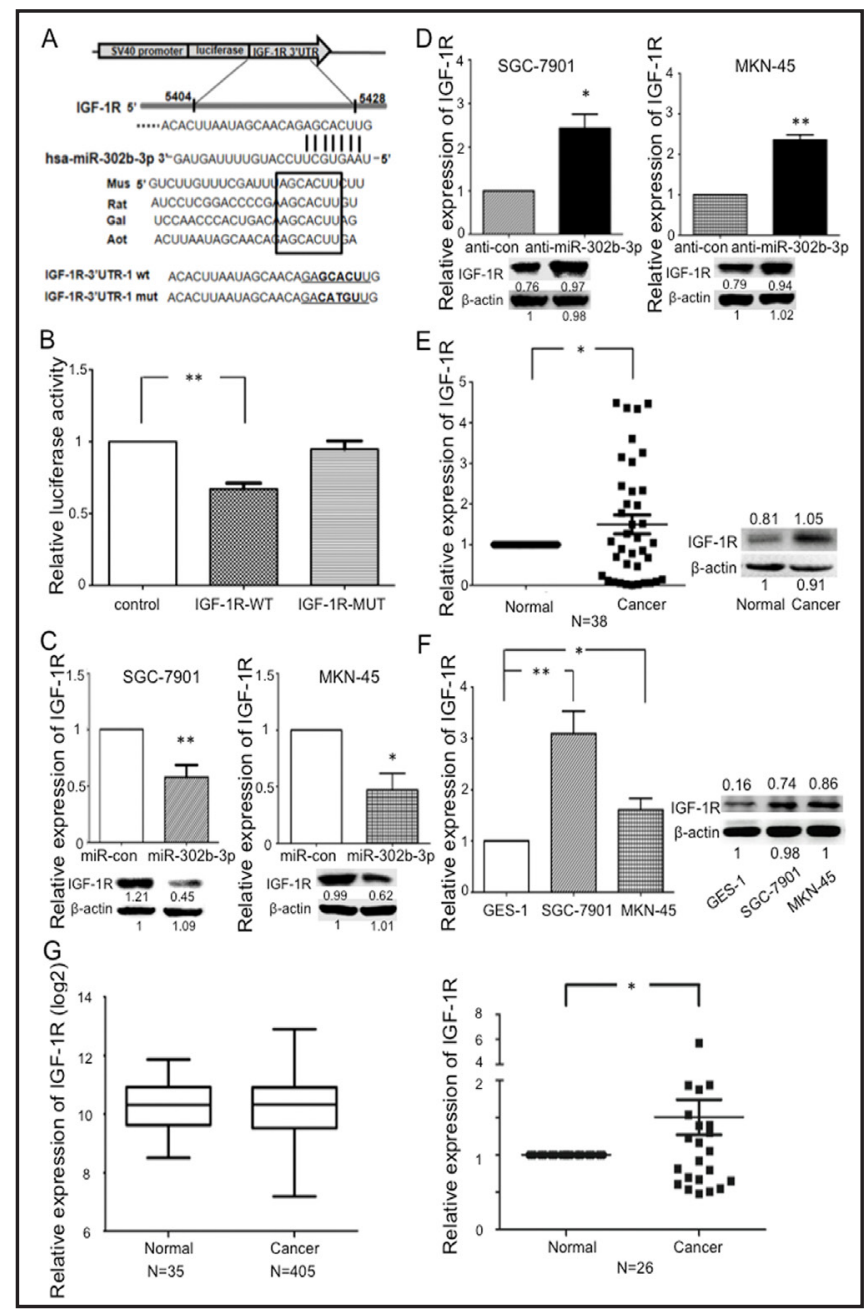

compared to the mut-type and control (Fig. 2B). In addition, we found that overexpression of miR-302b-3p suppressed the expression of IGF-1R at both the mRNA and protein levels (Fig. 2C). Additionally, transfection of the miR-302b-3p inhibitor increased the expression of IGF-1R in MKN-45 and SGC-7901 cells (Fig. 2D). We also examined the expression of IGF-1R in 38 paired tissue samples of GC and cancer cell lines. We found that IGF-1R expression was higher in both GC tissues (Fig. 2E) and cancer cell lines (Fig. 2F) compared to in their normal counterparts, which is consistent with the results of a high-throughput expression analysis of IGF-1R in the TCGA database (Fig. 2G). Taken together, the above data suggest that miR$302 \mathrm{~b}-3 \mathrm{p}$ directly regulates oncogene IGF-1R expression in GC cells.

\section{MiR-302b-3p decreases cell proliferation in SGC-7901/MKN-45 cells}

To explore the tumor-suppressor role of miR-302b-3p in GC, SGC-7901/MKN-45 cells were transfected with miR-302b-3p or controls. qRT-PCR was performed to examine the expression levels of miR-302b-3p after transfection with the miR-302b-3p expression vector or inhibitor and control. As expected, more than 40 -fold increases in miR-302b-3p expression was observed in both cell lines (Fig. 3A). To examine the inhibitory role of miR$302 b-3 p$ in GC cell growth, MTT and colony formation assay were conducted. The results showed that transient overexpression of miR-302b-3p led to inhibition of the growth of SGC$7901 /$ MKN-45 cells at 24, 48, and $72 \mathrm{~h}$ after transfection (Fig. 3B), resulting in fewer and smaller colonies compared to control cells (Fig. 3C). In contrast, the inhibitor of miR-302b$3 p$ significantly decreased expression (Fig. 3D), showing adverse effects in both cell lines (Fig. 3E/F). 


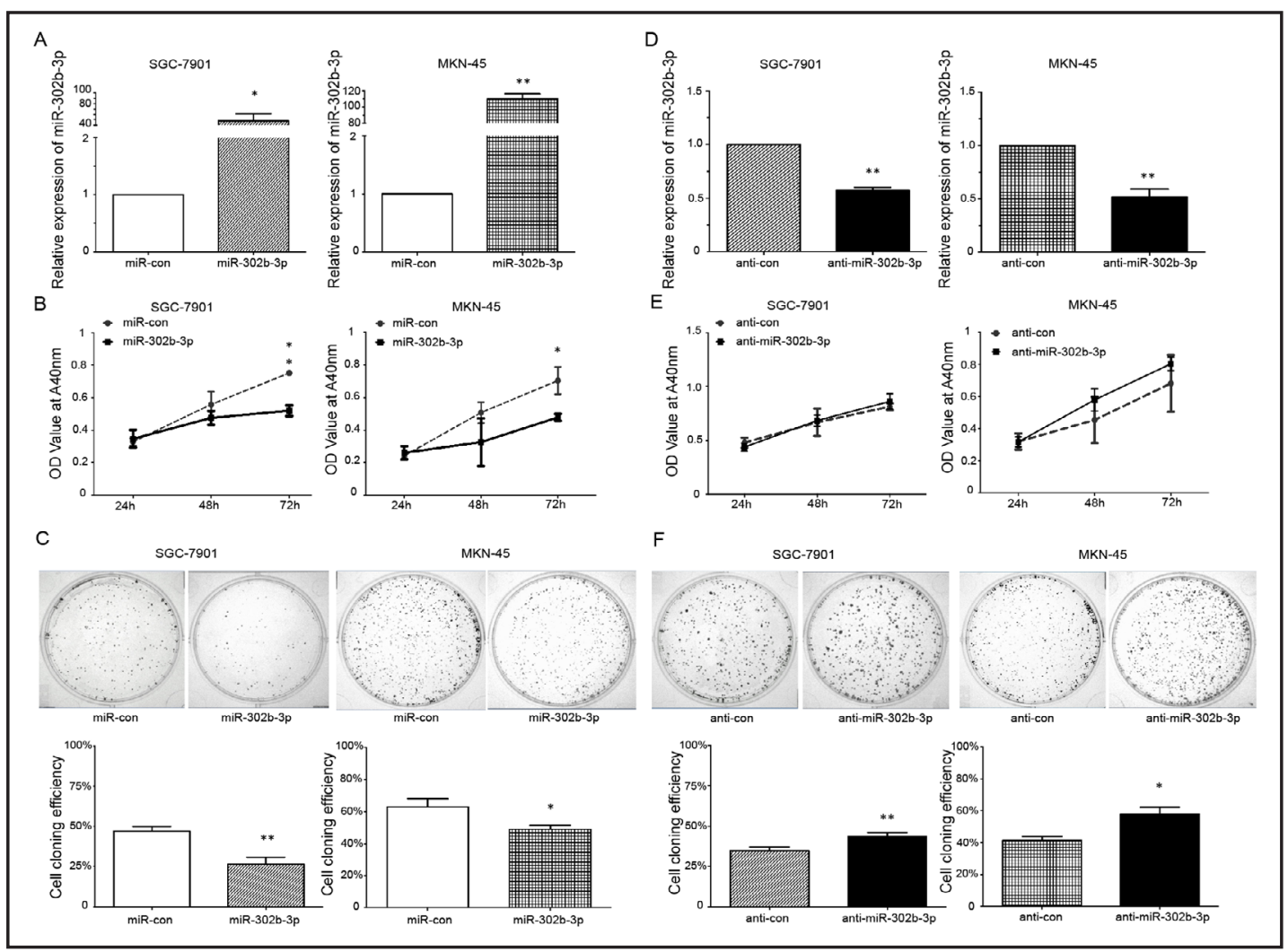

Fig. 3. MiR-302b-3p affects gastric cancer progression in vitro. (A) Enforced expression of miR-302b-3p were detected by qRT-PCR after transfect with miR-302b-3p vector. (B) The effects of miR-302b-3p on SGC$7901 /$ MKN-45 cell proliferation were determined by MTT assay at 24, 48 and $72 \mathrm{~h}$ respectively. (C) Representative results of colony formation of SGC-7901/MKN-45 cells after transfect with miR-302b-3p vector. (D) qRT-PCR analysis of miR-302b-3p expression in SGC-7901/MKN-45 cells transfected with anti-miR302b. (E) MTT assay was used to assay the effects of anti-miR-302b-3p on SGC-7901/MKN-45 cells. (F) Colony formation were performed in SGC-7901/MKN-45 cells after inhibition of miR-302b-3p. $\left(P^{*}<0.05\right.$, $\left.P^{* *}<0.01\right)$.

\section{MiR-302b-3p induces G1-S transition arrest by targeting IGF-1R-AKT pathway}

Next, to clarify whether miR-302b-3p-induced inhibition of cell proliferation resulted from a blocked cell-cycle checkpoint, SGC-7901/MKN-45 cells were transfected with the miR-302b-3p expression vector or control for $24 \mathrm{~h}$. Overexpression of miR-302b-3p resulted in marked accumulation of the G1-population in both GC cell lines (Fig. 4A), suggesting that miR-302b-3p blocked the G1-S transition. Reduced expression of miR-302b-3p by the inhibitor induced the cells to enter S-phase (Fig. 4B). To further investigate the molecular mechanisms of miR-302b-3p-induced cell proliferation repression, we detected the protein expression level of IGF-1R and its downstream regulators by western blotting. Our results showed that miR-302b-3p reduced the expression of IGF-1R protein and phosphorylation of p-AKT at serine 473, whereas protein expression of total AKT remained unchanged (Fig. 4C). Moreover, we analyzed the protein levels of related G1 regulators after overexpressing miR-302b-3p in SGC-7901 and MKN-45 cells by western blot analysis. Notably, miR-302b$3 p$ reduced the expression of cyclin D1 and CDK6, which are two essential regulators of the G1-S phase transition involved in AKT signaling pathway. These findings suggest that miR$302 \mathrm{~b}$ inhibits the proliferation of GC cells by affecting the IGF-1R/AKT pathways.

MiR-302b-3p induces apoptosis in SGC-7901/MKN-45 cells

In previous experiments, we found that overexpression of miR-302b-3p induced cell apoptosis. Compared with the control group, miR-302b-3p-transfected cells displayed 
Fig. 4. MiR-302b induces G1-S arrest in vitro through IGF-1R-AKT pathway. (A/B) Cell cycle was determined in SGC-7901/MKN-45 cells $24 \mathrm{~h}$ after transfected with miR-302b-3p or anti-miR-302b$3 p$ by flow cytometry. Histogram represented the percentages of cells in G0-G1, S and G2-M cell cycle phases. $\left(P^{*}<0.05\right)$. (C) Expression analysis for cell cycles regulators in the downstream of IGF-1R/AKT signaling pathway at $48 \mathrm{~h}$ after transfection by Western Blot.

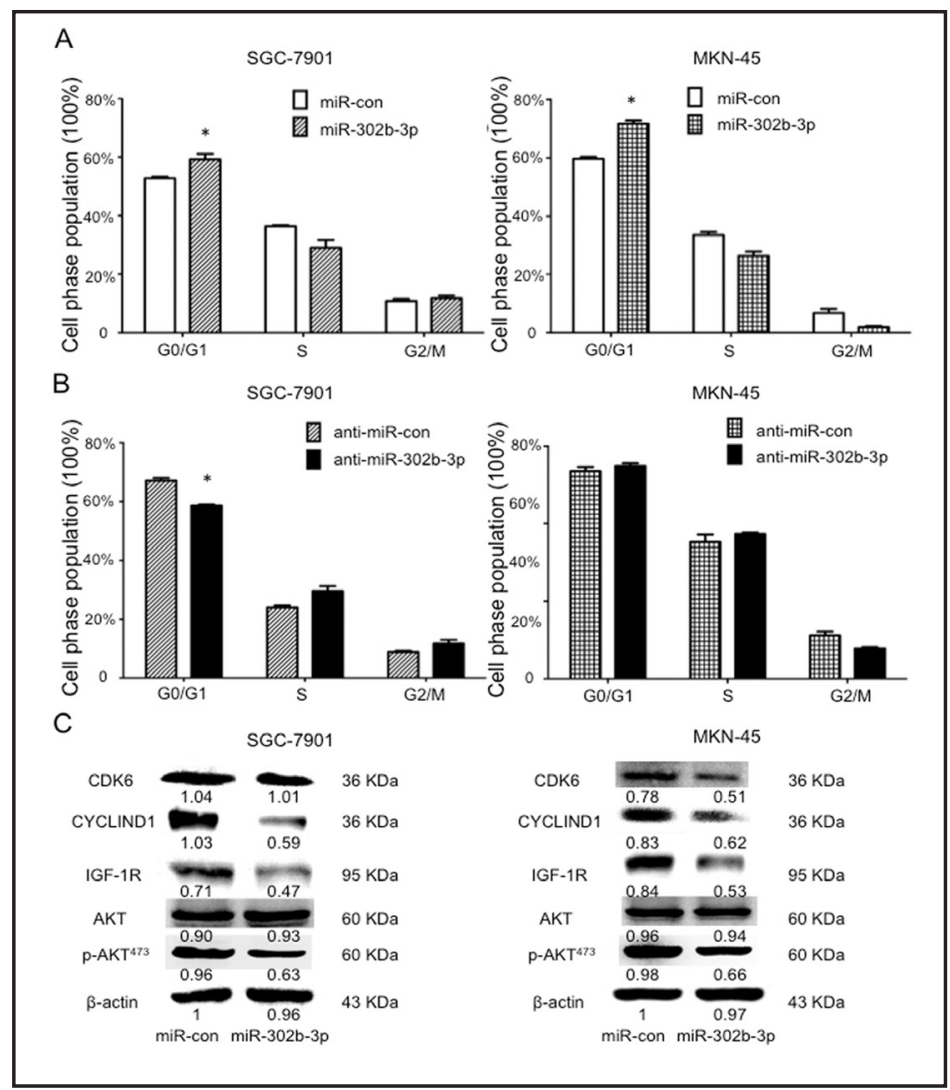

Fig. 5. MiR-302b-3p contributes to apoptosis of SGC-7901/MKN45 cells. (A/B) Cell apoptosis were detected by Annexin-V/Propidium iodide combined labeling flow cytometry in SGC-7901/MKN-45 cells $24 \mathrm{~h}$ after transfection. Apoptotic evaluation was calculated by the percentage of apoptotic cell number in total cell number. $\left(P^{*}<0.05, P^{* *}<0.01\right)$. (C) Expression of cell apoptosis related protein in the downstream of AKT signaling pathway were determined at $48 \mathrm{~h}$ after transfection by Western Blot.

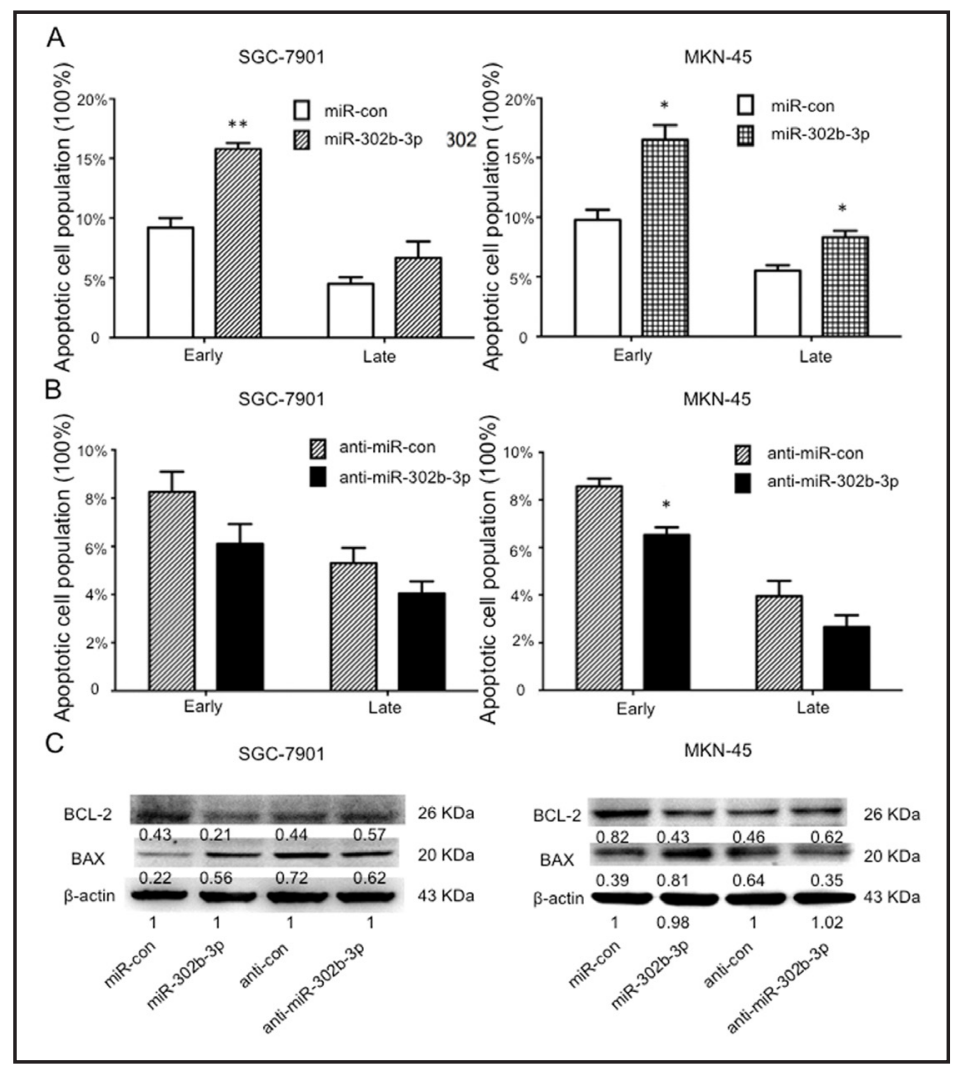

higher apoptotic rates (Fig. 5A), whereas the miR-302b-3p inhibitor showed lower apoptosis rates (Fig. 5B). Dysregulation of apoptosis is an important event in cancer, as it allows 

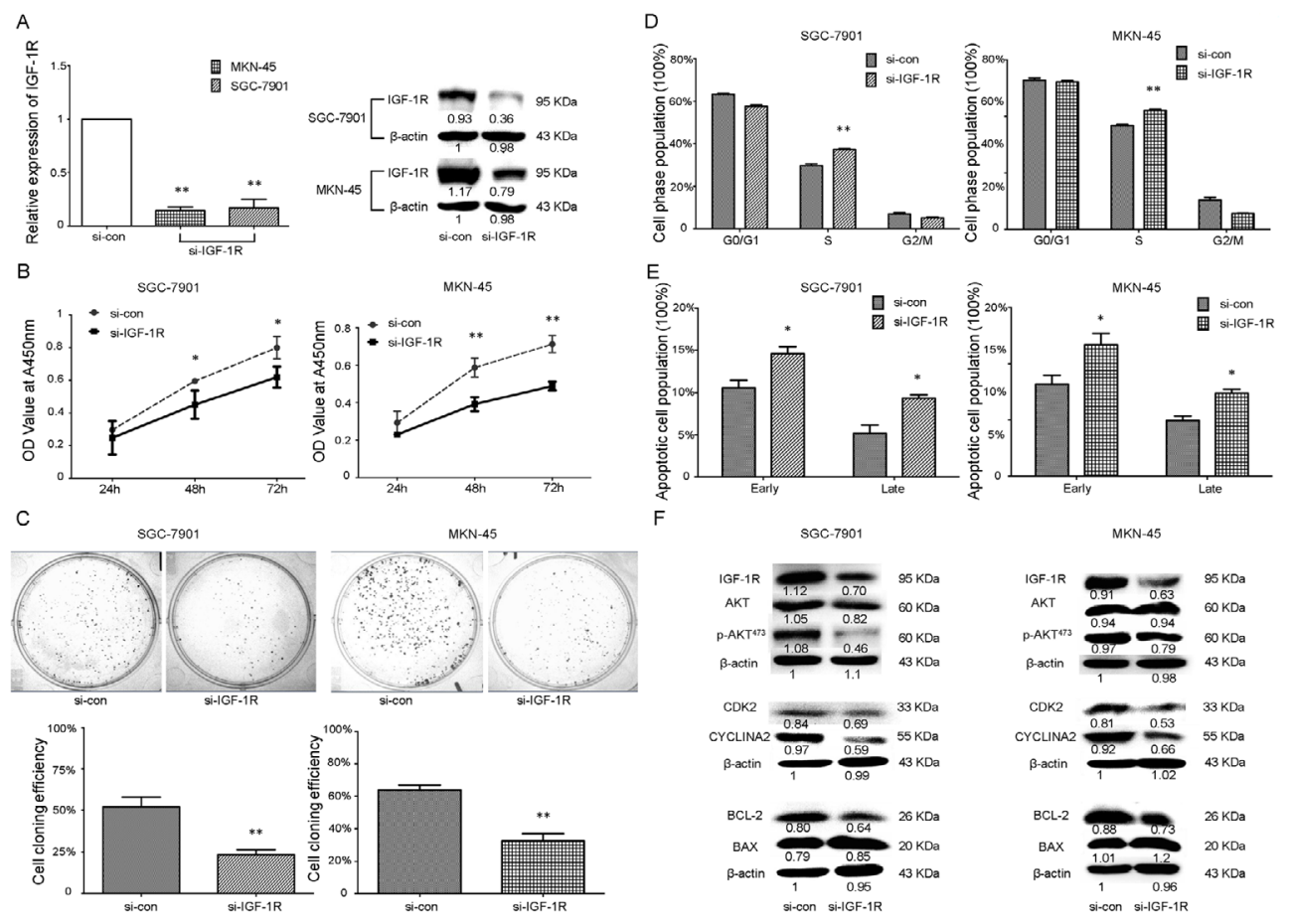

Fig. 6. Silence of IGF-1R suppresses gastric cancer cell growth and induces cell cycle transition arrest, cell apoptosis in SGC-7901/MKN-45 cells. (A) qRT-PCR (left portion) and Western Blot (right portion) were performed to determine the expression level of IGF-1R after transfection with IGF-1R siRNA. (B-E) MTT assay/ clone formation/cell cycle/cell apoptosis were performed to determine the impact of SGC-7901/MKN-45 cells treated with si-IGF-1R. (F) Expression analysis for IGF-1R/AKT signaling pathway at 48h after transfected with siRNA by Western Blot. $\left(P^{*}<0.05, P^{* *}<0.01\right)$.

genetically unstable cells to survive and accumulate additional mutations that eventually lead to tumorigenesis. We also focused on the apoptosis of GC cells and examined whether miR-302b-3p modified the downstream targets of the AKT pathway related to apoptosis. As a result, suppression of the IGF-1R-AKT pathway by miR-302b-3p promoted apoptosis by activating the proapoptotic protein Bax and inactivating the antiapoptotic protein $\mathrm{Bcl}-2$ (Fig. 5C).

Silencing of IGF-1R produces effects similar to those of miR-302b-3p overexpression in SGC-7901/MKN-45 cells

As described above, overexpression of miR-302b-3p affects cell proliferation, cycle, and apoptosis in GC cells. We also validated that IGF-1R is a direct target of miR-302b-3p. Therefore, we silenced IGF-1R expression by RNA interference to confirm that IGF-1R is involved in the antitumor effects caused by miR-302b-3p. Based on the mRNA and protein expression levels, IGF-1R was specifically knocked down by siRNA (Fig. 6A). Moreover, silencing of IGF-1R suppressed cell proliferation and induced S-G2 arrest and cell apoptosis (Fig. 6B-E), which followed the same trend as miR-302b-3p in SGC-7901/MKN-45 cells. These results were confirmed by protein expression analysis of the AKT pathway. As shown in Fig. 6F, the expression of IGF-1R protein and p-AKT were suppressed by si-IGF-1R, whereas total AKT remained unchanged. For cell-cycle regulation, si-IGF-1R reduced the expression of cyclin A2 and CDK2. Moreover, siRNA induced apoptosis by activating the proapoptotic protein Bax and inactivating the antiapoptotic protein Bcl-2. Based on these findings, miR$302 \mathrm{~b}-3 \mathrm{p}$ regulates GC progression by directly targeting IGF-1R through the Akt signaling pathway.

\section{KARGER}




\section{Cellular Physiology Cell Physiol Biochem 2017;42:1701-1711 \\ \begin{tabular}{l|l} 
DOI: 10.1159/000479419 & $\begin{array}{l}\text { O 2017 The Author(s). Published by S. Karger AG, Basel } \\
\text { www.karger.com/cpb }\end{array}$
\end{tabular} \\ Guo et al.: MiR-302b-3p Targets IGF-1R in Gastric Cancer SGC-7901/MKN-45 Cells}

\section{Discussion}

In recent decades, numerous studies have focused on the roles and functions of altered miRNAs in human GC. Approaches using miRNAs are considered to offer novel therapeutic opportunities for cancer [17]. MiR-302b belongs to the miR-302 cluster, which also contains miR-302a, -302a*, -302c, -302c*, -302d, -367, and -367*, and was first found in embryonic stem cells to help maintain stemness by regulating self-renewal and proliferation properties $[18,19]$. Most studies of the miR-302 cluster have focused on the maintenance of stemness and ability to reprogram somatic cells into induced pluripotent stem cells [20,21]. More recently, miR-302 was reported as a new tumor suppressor in several types of tumors. In cervical cancer cells, the miR-302-367 cluster was not endogenously expressed and its ectopic expression inhibited cell proliferation and tumor formation both in vivo and in vitro [22]. In addition, miR-302 was reported to inhibit human pluripotent stem cell tumorigenicity by enhancing multiple G1 phase arrest pathways [23]. However, few studies have examined miR-302b in GC and there are no data for miR-302b in TCGA database according to expression profile analysis. We found that miR-302b-3p was frequently downregulated in both GC tissues and cell lines, which is consistent with the results of recent studies [24]. Additionally, miR-302b expression led to inhibition of cell proliferation and clonogenicity and induced G1-S transition arrest as well as cell apoptosis in both GC SGC-7901 and MKN-45 cell lines. In contrast, loss of function studies were performed using anti-miR-302b-3p oligonucleotides to silence miR-302b-3p expression. The inhibitory effect revealed a clear contribution to the tumorigenicity of GC cells. These results indicate that miR-302b-3p is a novel tumorsuppressor miRNA in GC.

Furthermore, we identified IGF-1R as a direct target of miR-302b-3p. IGF-1R is a transmembrane receptor tyrosine kinase mainly activated by IGF1 or IGF2 through autocrine and paracrine pathways $[25,26]$ and is frequently overexpressed in human melanoma [27], head and neck cancer [28] and non-small cell lung cancer [29], suggesting that IGF-1R contributes to the initiation and progression of malignancies. In this study, through TCGA database analysis, we found that IGF-1R was overexpressed in 26 paired GC tissues. To confirm this, qRT-PCR and western blotting were conducted to detect IGF-1R expression in 38 paired GC tissues and 2 GC cell lines. The results showed that IGF-1R was overexpressed in GC. Further bioinformatics analysis revealed that miR-302b-3p has a well-matched binding site at the 3' UTR of IGF-1R. Dual-luciferase reporter assays demonstrated that miR-302b$3 p$ directly targeted IGF-1R by increasing IGF-1R mRNA expression and inhibiting IGF-1R translation.

IGF-1R has been reported to bind adaptor molecules such as insulin receptor substrates and Shc in an activated manner, which triggers multiple downstream signaling cascades, particularly phosphatidylinositol 3-kinase/AKT signaling pathways associated with resistance to apoptosis, increased cell growth, cell proliferation, and metabolism [3032]. However, it remains unclear which downstream targets are most critically related to IGF-1R induced hyper-activation of Akt for tumorigenesis in GC. In our study, IGF-1R was inhibited by the forced expression of miR-302b-3p and siRNA, which resulted in decreased phosphorylation of AKT. Previous studies showed that AKT phosphorylation is essential for cell cycle phase transition [33]. Considering the regulation of the cell cycle transition caused by miR-302b-3p and IGF-1R siRNA, we evaluated the effects on the AKT downstream cycle regulators cyclin D1/CDK6 and cyclin A2/CDK2 [34], which are key transcriptional factors in the G1-S and S-G2 transitions, respectively. The results revealed reduced expression levels of CDK6 and cyclin A2/CDK2 in SGC-7901/MKN-45 cells transfected with miR-302b-3p or si-IGF-1R. Although there were limited differences in cycle performance, which may be related to the multi-targeting effects of miRNA, further studies should examine the detailed mechanisms of these effects. Similarly, we found that the miR-302b-3p-induced IGF-1RAKT pathway plays an important role in regulating the Bcl-2 and Bax family, as previously described [35]. 


\section{Cellular Physiology Cell Physiol Biochem 2017;42:1701-1711

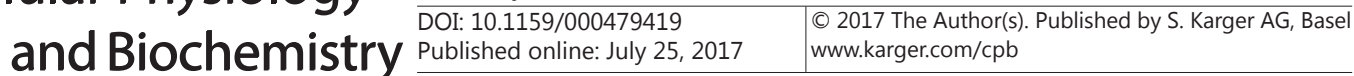 \\ Guo et al.: MiR-302b-3p Targets IGF-1R in Gastric Cancer SGC-7901/MKN-45 Cells}

In conclusion, we investigated the role of miR-302b-3p, its target gene IGF-1R, and their potential implications on the process of GC. These data suggest that miR-302b-3p is a novel tumor-suppressor that blocks the growth of GC cells through phosphatidylinositol 3-kinase/ AKT signaling pathways by targeting IGF-1R. Our findings reveal the function of miR-302b$3 p$ and indicate that miR-302b-3p may be a target for controlling GC.

\section{Acknowledgements}

This work was financially supported by Biomedical Sharing Platform Project of Xi'an Jiaotong University (2015FWPT-14), Shaanxi Province Science and Technology Projects (2014D75), National Natural Science Foundation of China (81660492), China Postdoctoral Science Foundation (2016M602801), and the Fundamental Research Funds for the Central Universities (0811/1191320073).

\section{Disclosure Statement}

The authors declare no conflict of interest.

\section{References}

1 Parkin DM, Bray F, Ferlay J, Pisani P: Global cancer statistics, 2002. CA Cancer J Clin 2005;55:74-108.

-2 Wang J, Wang Q, Liu H, Hu B, Zhou W, Cheng Y: MicroRNA expression and its implication for the diagnosis and therapeutic strategies of gastric cancer. Cancer lett 2010;297:137-143.

-3 Wu Q, Jin H, Yang Z, Luo G, Lu Y, Li K, Ren G, Su T, Pan Y, Feng B: MiR-150 promotes gastric cancer proliferation by negatively regulating the pro-apoptotic gene EGR2. Biochem Biophys Res Commun 2010;392:340-345.

Bartel DP: MicroRNAs: genomics, biogenesis, mechanism, and function. cell 2004;116:281-297.

Palma CA, Al Sheikha D, Lim TK, Bryant A, Vu TT, Jayaswal V, Ma DD: MicroRNA-155 as an inducer of apoptosis and cell differentiation in Acute Myeloid Leukaemia. Mol Cancer 2014;13:1.

6 Mulrane L, McGee SF, Gallagher WM, O'Connor DP: miRNA dysregulation in breast cancer. Cancer Res 2013;73:6554-6562.

7 Majid S, Dar AA, Saini S, Shahryari V, Arora S, Zaman MS, Chang I, Yamamura S, Tanaka Y, Chiyomaru T: miRNA-34b inhibits prostate cancer through demethylation, active chromatin modifications, and AKT pathways. Clin Cancer Res 2013;19:73-84.

-8 Suryawanshi S, Vlad AM, Lin H-M, Mantia-Smaldone G, Laskey R, Lee M, Lin Y, Donnellan N, Klein-Patel M, Lee T: Plasma microRNAs as novel biomarkers for endometriosis and endometriosis-associated ovarian cancer. Clin Cancer Res 2013;19:1213-1224.

-9 Guo B, Liu L, Yao J, Ma R, Chang D, Li Z, Song T, Huang C: miR-338-3p suppresses gastric cancer progression through a PTEN-AKT axis by targeting P-REX2a. Mol Cancer Res 2014;12:313-321.

10 Zhu P, Zhang J, Zhu J, Shi J, Zhu Q, Gao Y: MiR-429 induces gastric carcinoma cell apoptosis through Bcl-2. Cell Physiol Biochem 2015; 37:1572-1580.

11 Li X, Li H, Zhang R, Liu J, Liu J: MicroRNA-449a inhibits proliferation and induces apoptosis by directly repressing E2F3 in gastric cancer. Cell Physiol Biochem 2015; 35:2033-2042.

12 Wang D, Fan Z, Liu F, Zuo J: Hsa-miR-21 and Hsa-miR-29 in tissue as potential diagnostic and prognostic biomarkers for gastric cancer. Cell Physiol Biochem 2015; 37:1454-1462.

13 Wang Z, Liu M, Zhu H, Zhang W, He S, Hu C, Quan L, Bai J, Xu N: miR-106a is frequently upregulated in gastric cancer and inhibits the extrinsic apoptotic pathway by targeting FAS. Mol Carcinog 2013;52:634646. 


\section{Cellular Physiology Cell Physiol Biochem 2017;42:1701-1711 \begin{tabular}{l|l} 
DOI: 10.1159/000479419 2017 & $\begin{array}{l}\text { O 2017 The Author(s). Published by S. Karger AG, Basel } \\
\text { www.karger.com/cpb }\end{array}$
\end{tabular} \\ Guo et al.: MiR-302b-3p Targets IGF-1R in Gastric Cancer SGC-7901/MKN-45 Cells}

-14 Li J, Yu J, Zhang H, Wang B, Guo H, Bai J, Wang J, Dong Y, Zhao Y, Wang Y: Exosomes-Derived MiR-302b Suppresses Lung Cancer Cell Proliferation and Migration via TGFßRII Inhibition. Cell Physiol Biochem 2016; 38:1715-1726.

15 Wang L, Yao J, Zhang X, Guo B, Le X, Cubberly M, Li Z, Nan K, Song T, Huang C: miRNA-302b suppresses human hepatocellular carcinoma by targeting AKT2. Mol Cancer Res 2014;12:190-202.

-16 Zhu R, Yang Y, Tian Y, Bai J, Zhang X, Li X, Peng Z, He Y, Chen L, Pan Q: Ascl2 knockdown results in tumor growth arrest by miRNA-302b-related inhibition of colon cancer progenitor cells. PloS one 2012;7:e32170.

17 Xia L, Zhang D, Du R, Pan Y, Zhao L, Sun S, Hong L, Liu J, Fan D: miR-15b and miR-16 modulate multidrug resistance by targeting BCL2 in human gastric cancer cells. Int J Cancer 2008;123:372-379.

18 Ren J, Jin P, Wang E, Marincola FM, Stroncek DF: MicroRNA and gene expression patterns in the differentiation of human embryonic stem cells. J Transl Med 2009;7:20.

19 Suh M-R, Lee Y, Kim JY, Kim S-K, Moon S-H, Lee JY, Cha K-Y, Chung HM, Yoon HS, Moon SY: Human embryonic stem cells express a unique set of microRNAs. Dev Biol 2004;270:488-498.

20 Lin S-L, Chang DC, Chang-Lin S, Lin C-H, Wu DT, Chen DT, Ying S-Y: Mir-302 reprograms human skin cancer cells into a pluripotent ES-cell-like state. Rna 2008;14:2115-2124.

21 Lipchina I, Studer L, Betel D: The expanding role of miR-302-367 in pluripotency and reprogramming. Cell cycle 2012;11:1517-1523.

22 Cai N, Wang Y-D, Zheng P-S: The microRNA-302-367 cluster suppresses the proliferation of cervical carcinoma cells through the novel target AKT1. Rna 2013;19:85-95.

23 Lin S-L, Chang DC, Ying S-Y, Leu D, Wu DT: MicroRNA miR-302 inhibits the tumorigenecity of human pluripotent stem cells by coordinate suppression of the CDK2 and CDK4/6 cell cycle pathways. Cancer Res 2010;70:9473-9482.

24 Khalili M, Vasei M, Khalili D, Alimoghaddam K, Sadeghizadeh M, Mowla SJ: Downregulation of the Genes Involved in Reprogramming (SOX2, c-MYC, miR-302, miR-145, and P21) in Gastric Adenocarcinoma. J Gastrointest Cancer 2015;46:251-258.

25 Zhao X, Dou W, He L, Liang S, Tie J, Liu C, Li T, Lu Y, Mo P, Shi Y: MicroRNA-7 functions as an anti-metastatic microRNA in gastric cancer by targeting insulin-like growth factor-1 receptor. Oncogene 2013;32:13631372.

26 Janku F, Huang HJ, Angelo LS, Kurzrock R: A kinase-independent biological activity for insulin growth factor-1 receptor (IGF-1R): implications for inhibition of the IGF-1R signal. Oncotarget 2013;4:463.

27 Ramcharan RN, Aleksic T, Gao S, Tanner J, Darvill N, Bridges E, Asher R, Watson AJ, Margison GP, Repapi E: Inhibition of type 1 insulin-like growth factor receptor (IGF-1R) influences processing of replicationassociated DNA double-strand breaks (DSBs) and induces schedule-dependent sensitization of human melanoma to temozolomide (TMZ). Cancer Res 2014;74:1741-1741.

-28 Dale OT, Aleksic T, Shah KA, Han C, Mehanna H, Rapozo DC, Sheard JD, Goodyear P, Upile NS, Robinson M: IGF-1R expression is associated with HPV-negative status and adverse survival in head and neck squamous cell cancer. Carcinogenesis 2015;36:648-655.

-29 Peled N, Wynes MW, Ikeda N, Ohira T, Yoshida K, Qian J, Ilouze M, Brenner R, Kato Y, Mascaux C: Insulin-like growth factor-1 receptor (IGF-1R) as a biomarker for resistance to the tyrosine kinase inhibitor gefitinib in non-small cell lung cancer. Cell Oncol 2013;36:277-288.

30 Hay N, Sonenberg N: Upstream and downstream of mTOR. Genes Dev 2004;18:1926-1945.

-31 Chitnis MM, Yuen JS, Protheroe AS, Pollak M, Macaulay VM: The type 1 insulin-like growth factor receptor pathway. Clin Cancer Res 2008;14:6364-6370.

- 32 Johnson GL, Lapadat R: Mitogen-activated protein kinase pathways mediated by ERK, JNK, and p38 protein kinases. Science 2002;298:1911-1912.

33 Chang F, Lee J, Navolanic P, Steelman L, Shelton J, Blalock W, Franklin R, McCubrey J: Involvement of PI3K/ Akt pathway in cell cycle progression, apoptosis, and neoplastic transformation: a target for cancer chemotherapy. Leukemia 2003;17:590-603.

-34 Maddika S, Ande SR, Wiechec E, Hansen LL, Wesselborg S, Los M: Akt-mediated phosphorylation of CDK2 regulates its dual role in cell cycle progression and apoptosis. J Cell Sci 2008;121:979-988.

-35 Tsuruta F, Masuyama N, Gotoh Y: The phosphatidylinositol 3-kinase (PI3K)-Akt pathway suppresses Bax translocation to mitochondria. J Biol Chem 2002;277:14040-14047. 